This is a self-archived - parallel published version of this article in the publication archive of the University of Vaasa. It might differ from the original.

\title{
Why Is CryptoKitties (Not) Gambling?
}

Author(s): Serada, Alesja

Title: Why Is CryptoKitties (Not) Gambling?

Year: $\quad 2020$

Versio: $\quad$ AAM

Copyright (C) Alesja Serada | ACM 2020. This is the author's version of the work. It is posted here for your personal use. Not for redistribution. The definitive Version of Record was published in FDG '20 : International Conference on the Foundations of Digital Games, https://doi.org/10.1145/3402942.3402985.

\section{Please cite the original version:}

Serada, A. (2020). Why Is CryptoKitties (Not) Gambling?. In:

Yannakakis, G.N., Liapis, A., Kyburz, P., Volz, V., Khosmood, F. \& Lopes, P. (Eds.) FDG '2O : International Conference on the Foundations of Digital Games, 1-4.

https://doi.org/10.1145/3402942.3402985 


\title{
Why Is CryptoKitties (Not) Gambling?
}

Alesja Serada

School of Marketing and Communication, University of Vaasa, Vaasa, Finland, aserada@uwasa.fi

\begin{abstract}
CryptoKitties (Axiom Zen 2017) is a pioneering blockchain-based game that disrupts the 'classic game model' (Juul 2003; 2013) in a way that turns it into a gambling web application. As previous research has shown, its mechanics are almost exclusively based on chance (Scholten et al 2019), and the rest is mostly speculation with game assets (Lee, Yoo and Jang 2019). This raises the question whether this game requires any skill, such as strategic planning. In my case study, I revisit the game system and perform a practice of playing it to differentiate between unpredictable (or "aleatory", as in Johnson 2018) and decision-making points in the game. I argue that mapping the journey of a player should complement analysis of the game system to assess the balance between skill and chance in a better-informed manner.
\end{abstract}

\section{KEYWORDS}

Blockchain, online games, online gambling, Ethereum, video game ethics, game economies, open market.

\section{1 'Crypto Games' as the Latest Form of Gambling}

Gambling typically means wagering something of value on an event with an unpredictable outcome (Derevensky \& Griffith 2019). Uncertainty of outcome has always been present in games (Costikyan 2013; Johnson 2018), which also means that playing games has always invited manifold forms of gambling and betting. Most lately, chance mechanics in digital games and betting practices around them have led to the "gamblification" of sports and competitive video games (Macey and Hamari 2018).

Gambling has undergone several circles of marginalization and normalization in modernized societies (Caillois 1961). It is commonly agreed that "luck is seen often as a central way to make games fair" (Johnson 2018, 97). 'Democratic' games of chance provide equal opportunities to all participants, regardless of their skill and social status. Recent development of blockchain technologies and their application to games provide an opportunity to present game algorithms as transparent and even more 'fair' (Kraft 2019). However, such games are often criticized for lack of the actual gameplay and speculative tendencies (Lee, Yoo and Jang 2019), as well as for their close ties to gambling. In general, gambling can be detrimental to the wellbeing of players and to economies in general (Roger Caillois even labels it "the cancer of economies").

One of the best known blockchain-based games bases on chance mechanics is CryptoKitties, a browser game on the Ethereum platform published by Axiom Zen in 2017 \cite\{chohan_leisures_2017\}. Similar mechanics are present in many pet breeding simulators, such as Ovipets (IDZTECH 2011) and Dragon City (Social Point 2012), not to mention the Pokemon franchise. The principal difference is the fee paid to breed virtual pets $(0.008 \mathrm{ETH}$ for the most time of the game's existence). This fee is paid to execute a 'smart contract' that eventually leads to the birth of a new 'kitty' with a randomly assigned unique set of attributes, such as shape and color of its body parts. A combination of two attributes may result in a 'mutation', when a new attribute of a higher level presents itself: this may increase the 'kitty's' value. 'Kitties' are unique non-fungible Ethereum tokens kept in an Ethereum wallet (usually Metamask or the company's own wallet Dapper). Players can breed, sell, and buy 'kitties', which constitutes three meaningful choices available within the game system. There is also free play unrestrained by game rules; it happens on external platforms for trading and communication (OpenSea, Discord, Twitter and other less used digital spaces).

\section{Skills and Strategic Goals in CryptoKitties}

Are CryptoKitties gambling? Based on a set of criteria derived from Griffiths (Derevensky and Griffiths 2019, 633), Scholten et al (2019) conclude that CryptoKitties, as well as other Ethereum-based games, fit the definition of gambling and should be supervised as such (2019). However, what else is there, apart from unpredictability? Game designers commonly assess games based on prevalence of either chance or skill - an established division to which Jesper Juul also adds the third mechanic of labor (Juul 2013). Labor, in the 
case of blockchain games, is performed by miners who enable transactions and calculation of the attributes of new tokens on the blockchain. Next, we will look at applications of skill in the game.

The game can be described as a player's journey between 'aleatory' points (Johnson 2018) - unpredictable events that change the course of the game - and decision-making points such as choosing partners for breeding. The act of breeding that follows is a typical 'aleatory' point with an uncertain outcome. The probability of a desirable mutation is approximately $7 \%$ or less for the highest-level attributes. The probability of inheriting an attribute already present in parents is much higher, but is still not certain, as it depends on their complete genetic makeup: to put it simply, eight instances of 'genes' coded on blockchain are combined and randomized for each attribute. As in the updated definition of games of luck by Johnson, "player actions do not affect the outcome" (Johnson 2018, 95), and the main game mechanic does not differ much from a slot machine in this regard at this particular point, as Scholten et al. observe (2020).

As an additional technique of engagement, the game uses a well-known mechanic of completing a collection, widely used in playable media from children's sticker books to, again, Pokémon. The "sticker book" of the game is the Cat Codex - a web page with that represent every possible attribute in the game (Figure 1). After mastering the game system and learning genealogy and relative scarcity of different genes and attributes, the player might feel the urge to complete their collection of 'kitties' in the same way a jigsaw puzzle is completed. Contrary to Juul's understanding of a game, this final goal is optional; Juul conceptualizes the same urge in A Casual Revolution as "the satisfaction of moving the final piece, of finishing the puzzle" (Juul 2012, 2).

CryptoKitties deviates from the commonly acknowledged understanding of a game (Juul 2003, 2012, 2013) in one crucial aspect: the game does not have a final goal. It shares this feature with sandbox games and social games such as farming games and pet breeding simulators, but also, World of Warcraft and other roleplaying games (Costikyan 2013). Deciphered from the transparent design and the code of the game system, as initially programmed in 'smart contracts', the ultimate goal of the game would be to breed "the Queen Bee": a kitty which attributes are all of the highest level. As the game system itself is static - in fact, nothing moves or changes during the play, apart from new 'kitties' coming into existence and maybe changing their owners, - the game will not end for any player after breeding the Queen Bee, and this event will not influence any other element of the game. Players, who are free at goal setting, may not even acknowledge this possibility; it is a conscious decision they make themselves based on their own analytical skills applied to the game system or communication with fellow players. The Queen Bee can be bred any number of times, and each token will be unique and of high value for other players, although its value will diminish if thousands of similar tokens are created, which is highly improbable but generally possible.

\section{How CryptoKitties Are Actually Played}

As stated previously, the game only affords three actions: buying, selling and breeding (creating new digital 'kitties'). When focusing solely on these actions, gambling (Scholten et al 2019) and speculation (Lee, Yoo and Jang 2019) can be observed: cryptocurrency is invested in breeding 'kitties' in hope to randomly obtain a valuable one, and then players trade them on speculative open markets. These actions are recorded in the immutable ledger of a blockchain-based game, and researchers base their conclusions on their analysis of these records. However, one cannot fully understand what is happening in the game from "digital traces" and descriptions of the game. In this section, I will describe one game session of playing CryptoKitties as a performative practice, which will include both 'aleatory points' and decision-making. This is my own game session; although it relies on the official rules of the game, other players may play in different ways and set different goals.

Owing much to the collectible games genre, CryptoKitties encourage their players to get at least one 'kitty' with each existing attribute by filling the Cat Codex, as can be seen in Figure 1. The game does not reward players for it, it just feels like 'completion' (Juul 2012), which places this type of reward outside of the scope of strictly ludological approach. Many players of the game complete the Cat Codex - collect a "complete genome", as it is called in the community: this quest is not particularly difficult, as it is only a matter of the player's budget. After a player clicks on the blank space in the Cat Codex, the game redirects them to the Marketplace where they can purchase any kitty with any particular trait in two clicks for a price below the cost of its breeding. 
A more challenging approach is to fill the Cat Codex with kitties that have Gems - this means, one of the first 500 kitties who acquired a certain attribute as a result of a mutation. The total Gem count is displayed in the codex with the multipurpose Chrome extension CKBox, a commonly used, community-built interface layer that provides players with complete information on the game system. There are no 'leaderboards' in the game itself, but a player can check their rank in the game based on the Gem count at another external service, KittyHelper. Again, the Gem count can be increased by simply buying more kitties freely available on the market. 'Kitties' with Gems are more expensive - the lowest level Gem, the blue Lapis, raises the kitty's price somewhere around ETH 0,01 or higher (often still very close to the net cost of breeding it). For a not particularly wealthy player, it provides an interesting challenge (as in the academic understanding of a game) to occasionally fetch Lapis kitties priced below ETH 0,01 on the market (although a bot would still do it better, and such bots exist). In my case, it is more of an aesthetic challenge to complete the set with the kitties I personally prefer for no other rational reason than aesthetic pleasure.

As it can be seen in the Figure 1, the only attribute I am missing in the Wild Element set is "foghornpawhorn". This relatively scarce attribute looks like a cock's tail on a 'kitty'. There are two common ways to obtain such a kitty: to buy it on the market or to breed one yourself.

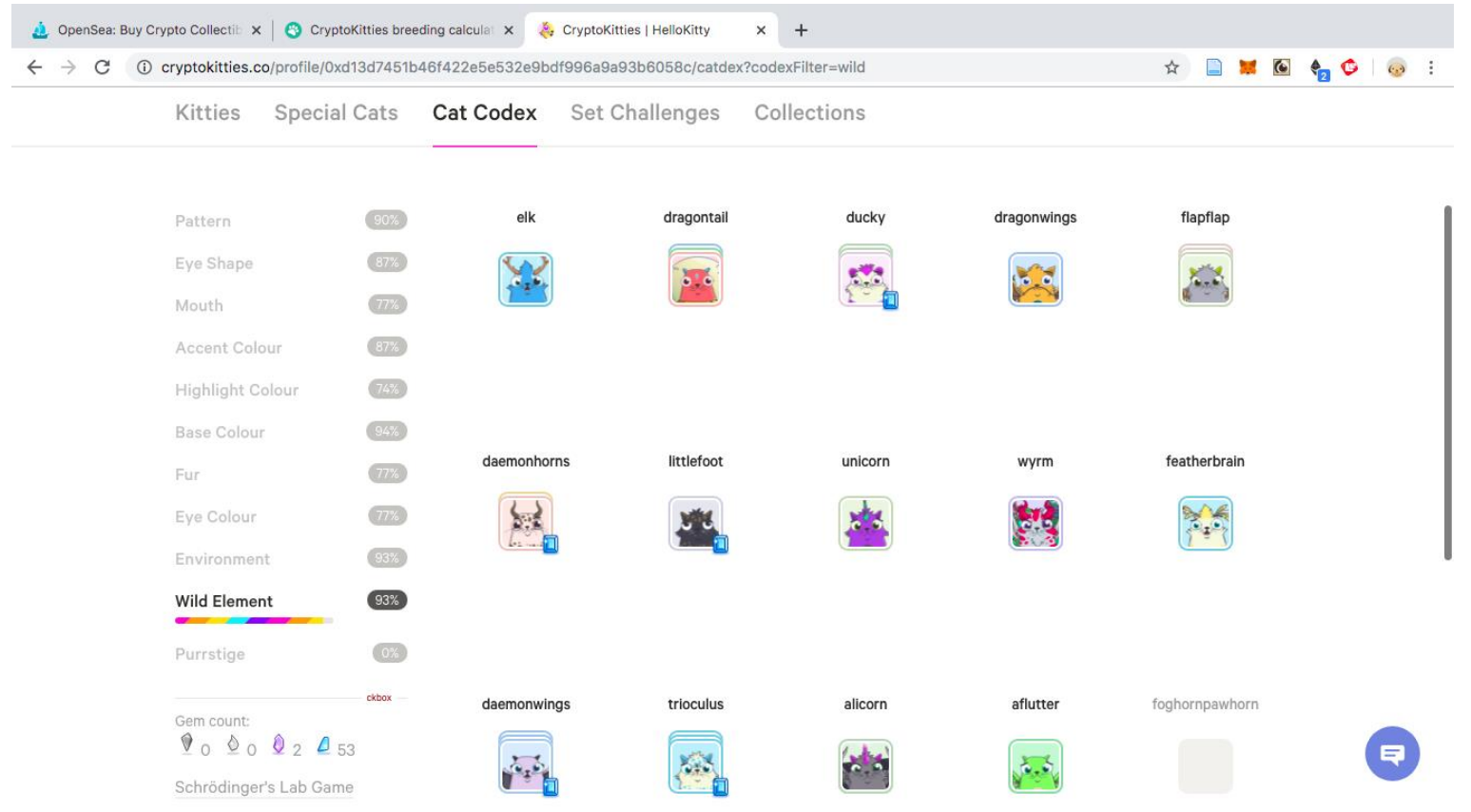

Figure 1: My Cat Codex

As I learned from sources like CKBox, KittyHelper among other sources, I need a Featherbrain and a Daemongwings to breed a unique Lapis Foghornpawhorn - the type of kitty I was missing from my desired set. Its generation also matters, as it drastically influences the price of a kitty - since rarer first generation kitties are valued at a much higher price on the market than latter generation kitties. I have a Generation 3 Lapis Featherbrain already, which is a relatively valuable kitty - it is reasonably rare, low generation, and I bought it for just $0.01 \mathrm{ETH}$, which I perceive it as cheap. My Daemonwings kitties are Generation 5 or older, which makes them an unsuitable pair for a Generation 3 Featherbrain, because offspring from Generation 3 and Generation 5 kitties would be Generation 6 - too much to be of value. I turn to the Marketplace to look for a Generation 3 Daemonwings that I could breed with for not too much ETH. The resulting kitty would be Generation 4, which is somehow valuable and probably can even sell with profit.

After I find a suitable candidate, by turning to KittyHelper, I learn that the chance to get a Foghornpawhorn mutation from two kitties with appropriate dominant genes is around $14 \%$. This is the standard fixed probability of a level 3 mutation under these conditions. Probability of the highest-level mutation is around $7 \%$. The probability may be higher if the "parents" have more than one required "gene" out of four, but this 
is very rare. Kitties with all four identical genes for one specific attribute are called "pure", and they are a moderately valuable collectible in some contexts.

Even though my actual chances to get a valuable kitty are low, I still decide to take the risk and breed with this kitty for $0,009 \mathrm{ETH}$, a fee which goes to its owner, plus a transaction fee of 0,008ETH, which I pay to 'miners' to generate a random new kitty. I also hope that the kitty will inherit the environment attribute MyParade, because, based on my previous experience, kitties with interesting environments tend to sell well even if they are of a higher generation (high-level attributes are valuable, but high-generation kitties are valueless). There are two more mutations possible with a 14\% probability: Hacker eyes and Splat pattern. I am not going for them specifically, but it still would be nice to get any of them, as it would usually raise the price of a kitty above the cost for its creation. Unfortunately, the resulting kitty has no promising attributes whatsoever, which would be prized in the marketplace, and can only be sold at a very low 'floor price'. For me, it means the loss of the most of $0,017 \mathrm{ETH}$, which is not that much, but still equals to around a quarter of a US dollar at the time of playing.

Let us return to my initial goal of completing the Wild Element collection. I still have some Ether from my previous, more successful operations, in my crypt wallet Metamask, so I go to the Marketplace again and find a Foghornpawhorn that I like. In addition to the Foghornpawhorn attribute, this kitty is depicted as sitting in a box, which is another attribute, the Tinybox environment. As a result, the kitty has one peculiar feature: its cock's tail is not visible, because the box environment covers it. It also has a Lapis gem for the mutation of the pattern Splat, which adds to its value on the market. I pay 0.025ETH for it, which is not cheap, by my standards, but it leaves me satisfied, as I believe that the sum of the quality of this kitty makes it "more unique" than a regular one. My Wild Element collection is now complete.

\section{Conclusion}

Like other pet breeding simulators, CryptoKitties does not set explicit goals for the player. Like a slot machine, it requires input of small sums of the cryptocurrency Ether to generate new 'kitties' of random value. This part of the game experience is an example of almost pure 'alea' (Caillois 1961) - a game of chance, owing its appeal to unpredictability of gameplay (Johnson 2018).

In additional to this gambling-like experience, players can set their own goals and make decisions only partially afforded or assisted by the official game system. With my analysis, I have demonstrated how mechanics of chance in the game are complemented by strategic breeding and trading. These activities require a very specific knowledge of the game and sometimes a skillset of a professional cryptocurrency trader. Players use a number of add-ons and third party resources such as CKBox and KittyHelper to quickly obtain important information about attributes of 'kitties' that is missing in the official interface. However, these skills of playing a 'crypto game' are rarely rewarded: success is still predominately based on chance, and the marketplace remains unpredictable.

Greg Costikyan describes tic-tac-toe as an example of a game without uncertainty (Costikyan 2013). In certain tasks, there is little uncertainty in the outcome of CryptoKitties for a player with enough resources: such player can complete almost any goal in the game by simply investing more Ether. This is where the gambling aspect becomes risky if the player is not able to stop transferring Ether into the 'kitty slot machine'. Yet, there is still enough strategic leeway between easily observable 'aleatory points' for a game of skill. Meaningful practices of playing the game emerge from deliberate choices of its players, their exploratory thrust or, in my described case, 'the urge of completion'. By combining both gambling-like and strategic elements, CryptoKitties has gamified the Ethereum platform, which has made it a noticeable factor in adoption of blockchain technologies.

\section{REFERENCES}

Caillois, Roger. 1961. Man, Play, and Games. University of Illinois Press.

Chohan, Usman W. 2017. The Leisures of Blockchains: Exploratory Analysis (December 4). Available at SSRN: https://ssrn.com/abstract=3084411 or http://dx.doi.org/10.2139/ssrn.3084411

Costikyan, Greg. 2013. Uncertainty in Games. MIT Press.

CryptoKitties. 2017. Online game. Axiom Zen. Available at www.cryptokitties.com. 
Derevensky, Jeffrey L., and Mark D. Griffiths. 2019. Convergence between gambling and gaming: does the gambling and gaming industry have a responsibility in protecting the consumer? In Gaming Law Review 23, no. 9 (November 1): 633-39. https://doi.org/10.1089/glr2.2019.2397.

Dragon City. 2012. Online game. Social Point.

Griffiths, Mark D. 2019. Loot Box Buying among Adolescent Gamers: A Cause for Concern? In Education and Health. Volume 37, Number 3.

Jaehwan Lee, Byungjoon Yoo, Moonkyoung Jang. 2019. Is a Blockchain-Based Game a Game for Fun, or Is It a Tool for Speculation? An Empirical Analysis of Player Behavior in Crypokitties. In The Ecosystem of e-Business: Technologies, Stakeholders, and Connections. Lecture Notes in Business Information Processing, vol 357. Springer, Cham. DOI: https://doi.org/10.1007/978-3-030-22784-5_14

Jesper Juul. 2013. The Art of Failure: An Essay on the Pain of Playing Video Games. The MIT Press.

Johnson Mark R.. 2018. The Unpredictability of Gameplay. Bloomsbury Academic, London.

Juul, Jesper. 2003. The Game, the Player, the World: Looking for a Heart of Gameness. In Level Up: Digital Games Research Conference Proceedings, edited by Marinka Copier and Joost Raessens, 30-45. Utrecht: Utrecht University, 2003.

Juul, Jesper. 2012. A Casual Revolution. MIT Press.

Kraft, Daniel. 2019. Game-Theoretic Randomness for Blockchain Games. In ArXiv:1901.06285 [Cs, Math], January 18. http://arxiv.org/abs/1901.06285.

Macey, Joseph, and Juho Hamari. 2018. Investigating Relationships between Video Gaming, Spectating Esports, and Gambling. In Computers in Human Behavior 80 (March): 344-53. https://doi.org/10.1016/j.chb.2017.11.027.

OpenSea. 2019. Digital marketplace. Available at https://opensea.io.

Ovipets. 2011. Online game. IDZTECH.

Scholten, Oliver James, Nathan Gerard Jayy Hughes, Sebastian Deterding, Anders Drachen, James Alfred Walker, and David Zendle. 2019. Ethereum Crypto-Games: Mechanics, Prevalence, and Gambling Similarities. In Proceedings of the Annual Symposium on Computer-Human Interaction in Play, 37989. Barcelona Spain: ACM, 2019. https://doi.org/10.1145/3311350.3347178. 